Ann. Sci. forest., 1971, 28 (2), 207-216.

\title{
NOTE POUR L'UTILISATION \\ DU PROGRAMME DE CALCULS \\ POUR LES INVENTAIRES FORESTIERS \\ PAR ÉCHANTILLONNAGE
}

\author{
J. BOUCHON, C. MILLIER et D. XEUXET \\ Station de Sylviculture et de Production, \\ Station de Biométrie, \\ Centre national de Recherches forestières, I.N.R.A., \\ 54 - Nancy \\ RÉSUMÉ
}

Les auteurs expliquent l'usage des imprimés et la manière de présenter les données pour l'utilisation d'un programme de calcul pour les inventaires forestiers par échantillonnage qu'ils ont eux-mêmes mis au point.

Un article paru récemment dans la Revue Forestière Française (Bouchon, Millier, XEuXET - 1970) de juillet-août 1970 présentait le programme de calculs poùr les inventaires forestiers par échantillonnage mis au point au C.N.R.F.: le présent article a pour but d'en faire une description technique plus détaillée.

\section{1. - BUT DU PROGRAMME}

A partir de données primaires recueillies sur le terrain, ce programme donne des estimations des éléments (volume par parcelle, accroissements en volume, etc...) nécessaires à l'établissement des procès-verbaux d'aménagement. Les calculs d'erreurs pernettant d'obtenir les intervalles de confiance reposent sur les lois de la statistique classique de l'échantillonnage au hasard d'ensembles homogènes, après stratification éventuellement.

\section{2. - PRÉSENTATION DES DONNÉES}

Les données relevées sur le terrain sur des imprimés à 80 colonnes tels que celui donné en exemple sont perforées sur des cartes. Le paquet de cartes présenté à l'ordinateur se compose de trois parties. 


\section{1. - Cartes paramètres initiales}

Les données figurant sur ces cartes sont générales à la forêt considérée. L'examen de leur contenu permettra de mieux en comprendre l'utilité.

Carte 1: c'est une carte définissant en code les organes d'entrée (par exemple la carte perforée) et de sortie (par exemple la machine imprimante). Cette carte est directement fournie par le service de calcul.

Cartes 2 à 4 : ces cartes contiennent le nom de la forêt qui servira de titre au moment de la sortie des résultats.

Carte 5 : c'est la «carte paramètres » proprement dite. Elle contient dans l'ordre :

- Colonnes 1-2: Une valeur LLL négative si les tarifs de cubage utilisés sont des fonctions (par exemple: $\mathrm{V}=\mathrm{a}+\mathrm{bD}^{2}$ ), positive ou nulle si les tarifs sont des tableaux.

- Colonnes 3-4: le nombre d'essences NBESS (inférieur à 11).

- Colonnes 5-6: un nombre MIESS positif ou nul si on désire que le nom des essences apparaisse en clair dans les résultats, négatif si on désire que le nom de l'essence demeure remplacé par un code.

Colonnes 9 à 24 : la suite des valeurs d'une variable indicée LNESS (I) qui correspond au nombre d'essences auxquelles on veut appliquer le ième tarif qui se trouve dans les cartes suivantes.

Ainsi, lorsque NBESS $=3$ si on veut appliquer un seul tarif, on aura LNESS $(1)=3$, tous les autres LNESS étant nuls. Par contre, si NBESS $=10$ et qu'on veut appliquer le premier tarif de cubage au 6 premières essences, le deuxième tarif à la septième essence, le troisième tarif aux huitième et neuvième essences et le quatrième tarif à la dernière essence, on aura :

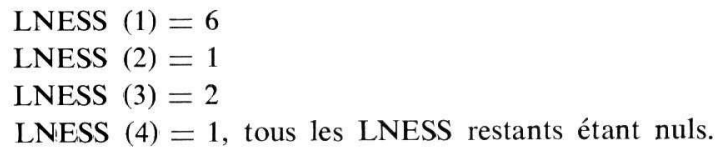

- Colonnes 29-30 et 31-32: le numéro de la catégorie de diamètre correspondant au maximum des «jeunes bois » et le numéro de la catégorie de diamètre correspondant au maximum des «bois moyens»; par exemple, si ces valeurs sont 3 et 7, les jeunes bois seront ceux dont le diamètre est inférieur ou égal à $22,5 \mathrm{~cm}$ et les vieux bois ceux dont le diamètre est supérieur à $42,5 \mathrm{~cm}$. (La première catégorie contient tous les arbres de diamètre inférieur à $12,5 \mathrm{~cm}$, les suivantes étant les catégories classiques de $5 \mathrm{~cm}=15,20,25, \ldots 125 \mathrm{~cm}$ ).

La figure 2 permet de constater que l'entier LLL n'a que 2 chiffres, que la zone des LNESS (I) n'a pas un dessin régulier, qu'il existe enfin un trou (col. 25-28) avant les zones réservées aux valeurs limites des classes de diamètres.

Cartes $(6,7)_{1},(6,7)_{2}, \ldots(6,7)_{i} \ldots$ : Ce sont les cartes «tarifs de cubage ». Ces cartes n'existent que si LLL $\geqslant 0$. Comme un tarif occupe deux cartes, il y aura autant de couples $(6,7)$ qu'il y a de LNESS (I) différents de zéro.

Exemple: Si on veut utiliser 2 tarifs sous forme de tableau, dans la carte 5 la valeur de LLL sera positive ou nulle, LNESS (1) et LNESS (2) étant différents de zéro. On aura deux couples de cartes $(6,7)$; la figure 1 montre comment se présenteraient les tarifs Algan $\mathrm{n}^{\circ} 11$ et $\mathrm{n}^{\circ} 14$.

Si l'on veut au contraire utiliser un tarif sous forme de fonction, par exemple :

$$
\mathrm{V}=-42,19+0,8357 \mathrm{D}^{2}
$$




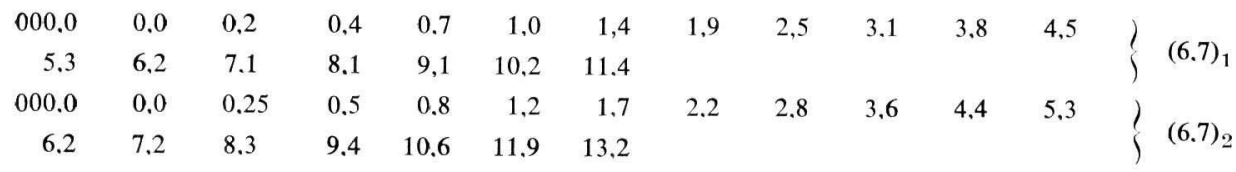

FIG. 1. - Les cartes $(6,7)_{1}$ et $(6,7)_{2}$ contiennent les tarifs Algan $n^{\circ} 11$ et 14 FIG. 1. - Cards numbered $(6.7)_{1},(6.7)_{2}$ contain Algan Volume Tables $n^{\circ} 11$ and 14

dans la carte 5, la valeur de LLL sera négative, et l'ensemble des cartes $(6,7)$ sera remplacé par une seule carte :

$$
\text { - 42,19 } \quad 0,0 \quad 0,8357
$$

Carte 8: Cette carte n'existe que si MESS $\geqslant 0$. Elle contient les noms des essences.

La figure 2 donne un exemple des cartes $\mathrm{n}^{\circ} 1$ à 8 .

Les noms des essences sont abrégés ; CHEN pour chêne, HETR pour hêtre, CHRM pour charme, FLLU pour feuillus divers.

\section{INVENTAIRE PAR ECHANTILLONNAGE FORET DE DARNEY}

032

040 $4000000 \quad 000$

$\begin{array}{llll}000.0 & 0.0 & 0.2 & 0.5\end{array}$

$\begin{array}{lllllll}006.1 & 7.2 & 8.3 & 9.4 & 10.7 & 12.0 & 13.4\end{array}$

$1.7 \quad 2.2$

\section{CHENHETRCHRMFLLU}

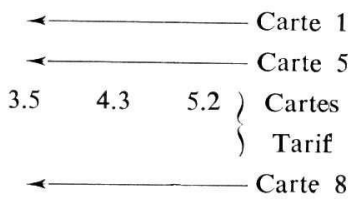

FIG. 2. - Cartes $n^{\circ} 1$ à 8. Chaque ligne correspond à une carte à 80 colonnes. Dans le cas présent il y a 4 essences auxquelles on applique le même tarif

Fig. 2. - Cards $n^{\circ} 1$ to 8 . To each ligne corresponds a punched card. In this example, we have 4 species which have the same volume table

\section{2. - Cartes inventaires proprement dites}

Ce sont les cartes perforées à partır des imprimés de terrain. L'ordre dans lequel elles doivent être présentées est rigoureux: les cartes d'une même série (au sens forestier du terme) doivent se suivre. A l'intérieur de chaque série les cartes de chaque affectation ou groupement de parcelles doivent se suivre de même que les cartes d'une mêmé parcèllé à l'intérieur de chacun de ces groupes de parcelles. Enfin les cartes relatives à chaque placette échantillon doivent se présenter dans l'ordre suivant:

- $n$ cartes inventaires, correspondant au nombre $n$ d'essences inventoriées dans la placette ; $n$ peut varier de 1 à NBESS ; s'il n'y a pas d'arbre mesurable dans la placette, une carte inventaire est quand même nécessaire : elle sera blanche à partir de la colonne 31 .

- 1 carte pour les 3 arbres échantillons;

- 1 carte pour la description de la placette.

On trouvera en annexe $n^{\circ} 1$ un exemple de fiche remplie sur le terrain pour une placette, avec les recommandations pour l'usage de ces imprimés.

\section{3. - Cartes de fermeture}

Il y en a 3. Elles déclanchent les calculs en fin de passage des données. Elles sont fournies par le bureau de calcul. 


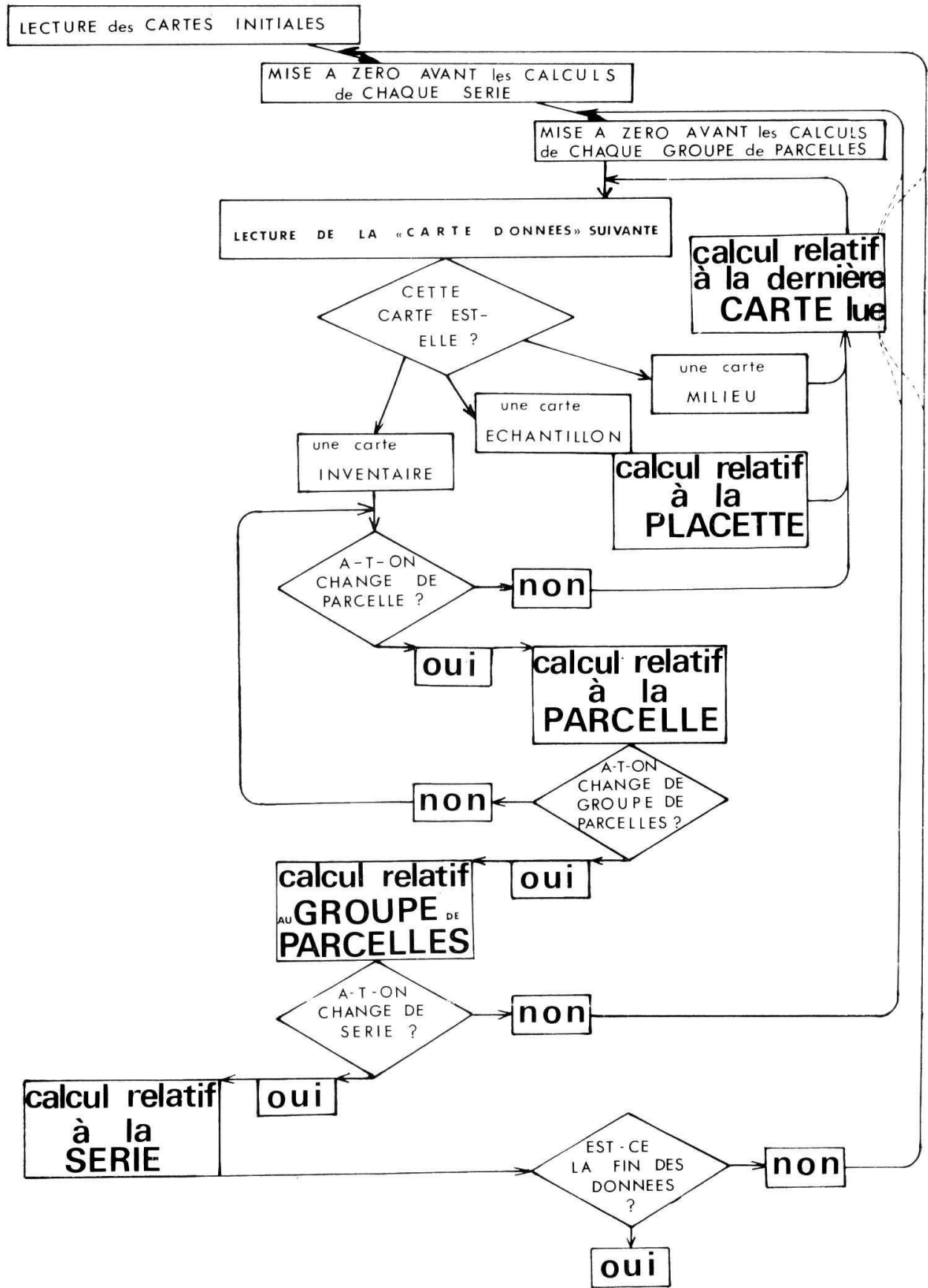

FIG. 3. - Schéma du programme de calcul

FIG. 3. - Flow chart for computer program 


\section{3. - ORGANISATION DU PROGRAMME}

Le schéma ci-joint (voir figure 3) explique le déroulement du programme; par souci de simplification ne figurent pas les tests de début de programme; ne figurent pas non plus les tests de fin de groupes de parcelles permettant à la suite des calculs y relatifs de revenir à la carte qui a déclanché le calcul : étant la première carte du groupe suivant elle doit être prise en compte, alors que le schéma simplifié ne l'indique pas.

On distingue principalement 5 boucles imbriquées qui correspondent aux 5 niveaux des calculs :

- dernière carte inventaire qui a été lue ;

- groupe de cartes inventaires: placette échantillon ;

- groupe de placettes échantillons : parcelles ;

- groupe de parcelles;

- serie de groupes de parcelles.

Ce programme écrit en FORTRAN a été mis au point sur IBM 1 130. La taille relativement modeste de cet ordinateur comparée à la quantité de variables nécessaires dans le programme a obligé le découpage en nombreux sous-programmes. Parmi eux, un seul est spécifique de la forêt traitée et doit être rédigé pour chaque inventaire: e'est la fonction MNEMO qui permet de passer des codes discontinus des essences de la forêt à une série d'indices continus variant de 1 à NBESS : un exemple de fonction MNEMO est donné en annexe 2 .

\section{4. - MISE EN CEUVRE DU PROGRAMME}

L'Institut National de la Recherche Agronomique n'est pas destiné à assurer l'exploitation des résultats des travaux qu'il entreprend: mais le programme est disponible sur simple demande à l'un des auteurs. A ce programme seront joints les FORMATS des cartes de données et quelques exemples de fonction MNEMO.

L'usage qui se généralise des ordinateurs demande à tous une reconversior: et un effort, ne serait-ce que celui de prospecter le marché pour trouver des organismes disposés à effectuer les calculs. Le gain de temps, d'argent et d'information qui en résulte n'en vaut-il pas la peine ?

Reçu pour publication en mars 1971.

\section{SUMMARY}

EXPLANATORY NOTE FOR THE USE OF AN AUTOMATICAL PROGRAM ON FOREST INVENTORIES MADE BY SAMPLING

The authors give explanations for the use of printed papers and presentation of data for the utilization of an automatical program on forest inventories.

\section{ZUSAMMENFASSUNG \\ ANWEISUNGEN FÜR DIE BENÜTZUNG EINES AUTOMATISCHEN RECHENPROGRAMMES IN FORSTLICHEN STICHPROBENINVENTUREN}

Die Autoren geben eine genaue Beschreibung der Aufnahmeformulare zur Benützung eines von ihnen ausgearbeiteten automatischen Rechenprogrammes bei forstlichen Stichprobeninventuren.

\section{RÉFÉRENCES BIBLIOGRAPHIQUES}

J. Bouchon, C. Millier, D. Xeuxet. Programme de Calculs pour les inventaires forestiers par échantillonnage. Revue forestière française, XXII, 4, 1970, pp. 451-462. 


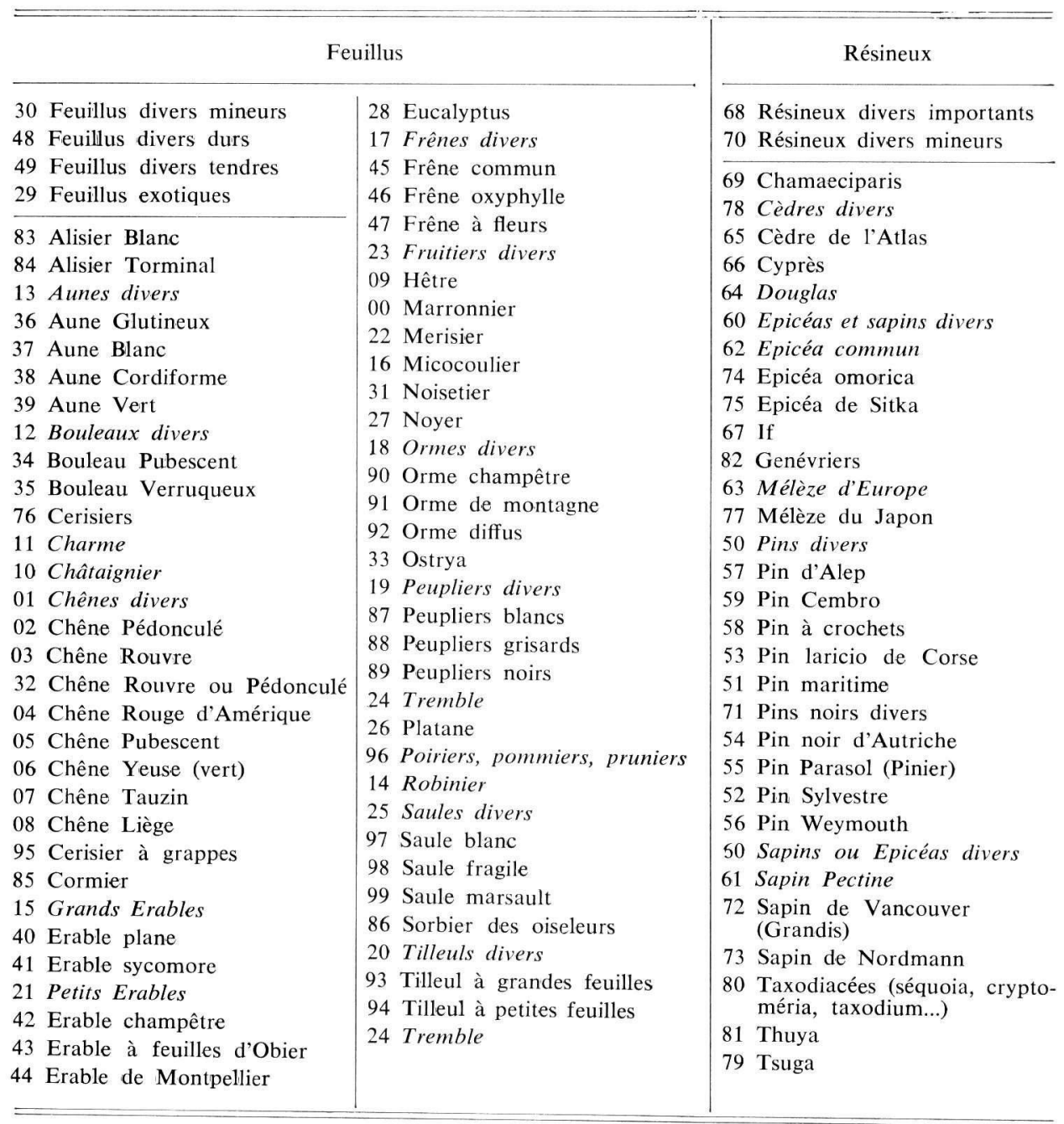




\section{ANNEXE 1 \\ INVENTAIRE PAR ÉCHANTILLONNAGE \\ RECOMMANDATIONS POUR L'USAGE DES IMPRIMÉS (FIG. 4)}

\section{1. - Généralités}

Chaque ligne à 80 colonnes représente une carte qui devra être perforée pour être utilisée par l'ordinateur. Il est done important de ne pas se tromper de colonne pour la transcription d'une mesure et de ne mettre qu'un chiffre dans chaque case. Dans chaque placette échantillon, trois modèles de cartes peuvent être remplis (cartes numérotées 7,8 ou 9 en $2^{\circ}$ colonne).

\section{2. - Colonnes 1 à 12}

Ces colonnes servent pour identifier la carte.

La colonne 1 reste vide.

La colonne 2 contient :

7 pour les cartes «inventaires»,

8 pour la carte «échantillon»,

9 pour la carte «milieu».

Les colonnes 3 à 12 sont identiques pour toutes les cartes d'une même placette.

Colonnes 3-4: deux derniers chiffres de l'année de la mesure.

Colonnes 5-6: $\mathrm{n}^{\circ}$ du département.

Colonnes 7 à $10: \mathrm{n}^{\circ}$ de la placette.

La colonne 11 n'est pas à remplir (elle le sera par le bureau de calcul) et ne sert qu'à distinguer éventuellement deux inventaires faits la même année dans le même département. Cependant, si, dans la colonne 12 , le $\mathrm{n}^{\circ}$ de la série dépasse 9, mettre le chiffre des dizaines dans la colonne 11 .

\section{3. - Carte 7 : «inventaire». Colonnes 13 à 80}

Ces cartes servent à faire l'inventaire de la placette. Il en sera rempli une par essence ou par groupe d'essences (par exemple, feuillus durs, feuillus tendres, etc...).

Colonne 13 : type de la forêt.

Colonne 14 : méthodes d'aménagement : pour le moment, ne pas remplir ces deux colonnes.

Colonne 15 : numéro d'affectation, ou de quartier, etc..., à l'intérieur de la série.

Colonne 16: regroupement de placettes, codé de 0 à 9 à la discrétion du gestionnaire, en fonction par exemple de critères écologiques, pédologiques, de relief, de régénération, etc... (facultatif).

Colonnes 17 à 20 : surface de la parcelle en ares.

Colonnes 21 à 23 : numéro de la parcelle dans laquelle se trouve le centre de la placette échantillon.

Colonnes 24-25: pente en degré.

Colonnes 26 à 30 : surface de la placette échantillon en $\frac{\mathrm{m}^{2}}{10}$. 


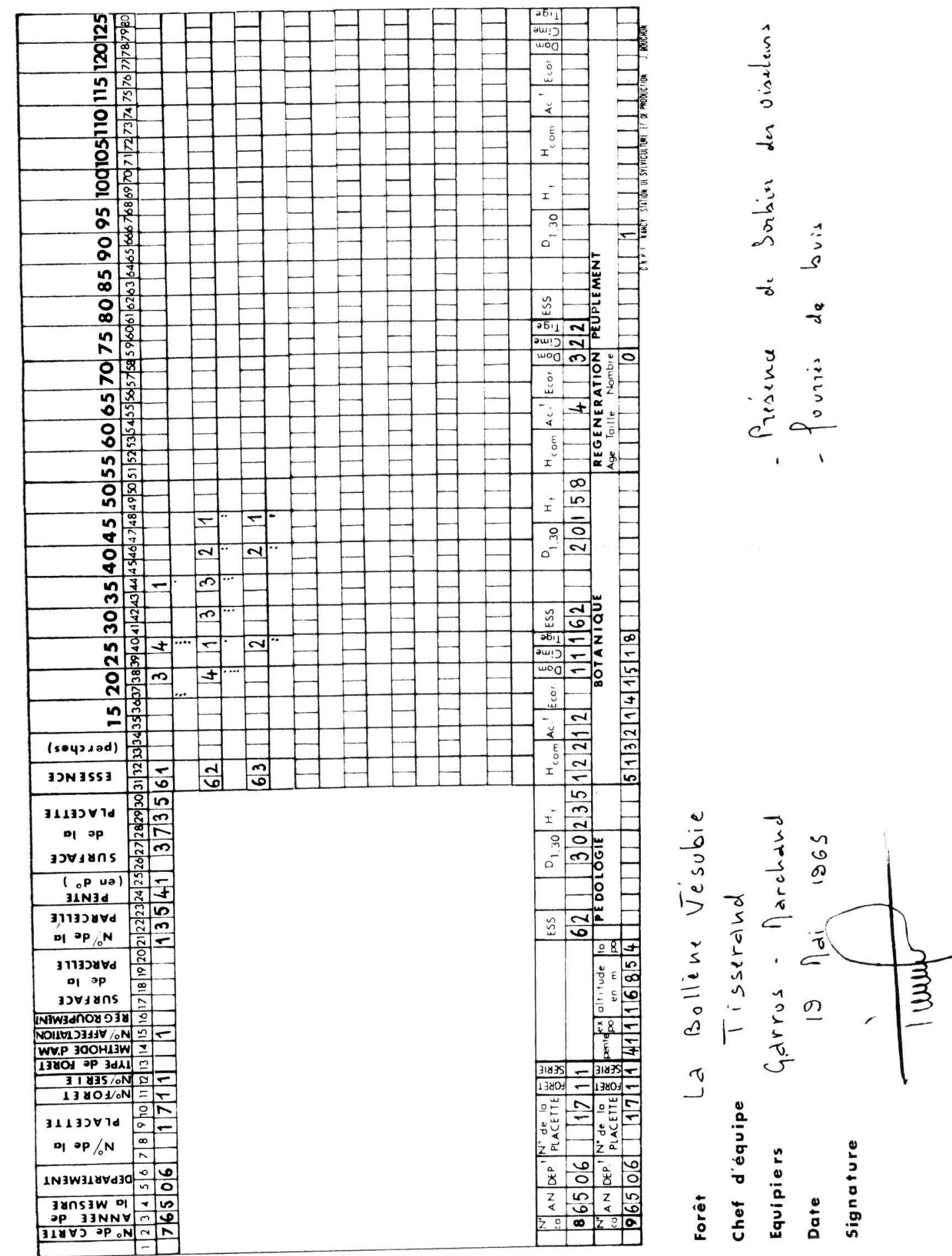

FIG. 4. - Imprimé de terrain pour la transcription des mesures FIG. 4. - Sheet for data transcription in the field 
Ainsi, une placette de 2,5 ares sera transcrite :

\begin{tabular}{|c|c|c|c|c|c|c|c|}
\hline Colonnes & 25 & 26 & 27 & 28 & 29 & 30 & 31 \\
\hline & & & 2 & 5 & 0 & 0 & \\
\hline & & & 2 & 5 & 0 & 0 & \\
\hline & & & 2 & 5 & 0 & 0 & \\
\hline
\end{tabular}

Colonnes 31-32 : Essence, voir le code joint.

Colonnes 33 à 80 : inventaire par catégories de diamètre (Les lignes suivant immédiatement chaque carte $\mathrm{n}^{\circ} 7$ servent éventuellement pour le pointage).

\section{4. - Carte 8: «échantillon». Colonnes 21 à 80}

Ces cartes correspondent à trois arbres échantillons au maximum, compris respectivement dans les colonnes 21 à 40,41 à 60,61 à 80 . Nous ne décrirons donc que les colonnes 21 à 40 .

Colonnes 21-22 : Essence, voir le code joint.

Colonnes 23-24: à la discrétion du gestionnaire.

Colonnes 23-24: à la discrétion du gestionnaire.

Colonnes 25-27 : diamètre à 1,30 en $\mathrm{cm}$ (compensé ou non).

Colonnes 28-30: hauteur totale en $\mathrm{dm}$.

Colonnes 31-33: hauteur commerciale en $\mathrm{dm}$.

Colonnes 34-35: accroissement sur le rayon à 1,30 $\mathrm{m}$ des dix dernières années en mm.

Colonnes 36-37 : épaisseur d'écorce à $1,30 \mathrm{~m}$ en $\mathrm{mm}$.

Colonne 38 : caractère de dominance (1 dominant, 2 codominants, 3 dominés).

Colonne 39: caractère de cime (1 plus large que la normale, 2 normalement développés, 3 étriqués).

Colonne 40 : caractère de la tige (à la discrétion du gestionnaire).

Toutes les mesures de la carte 8 sont facultatives.

\section{5. - Carte 9: «milieu». Colonnes 13 à 80}

Colonnes 13-14: pente en $\mathrm{d}^{\circ}$.

Colonne 15 : exposition $(1=$ N. $; 2=$ N.-E. $; 3=$ E. $; 4=$ S.-E. $; 5=$ S. ; $6=$ S.-W. ; $7=$ W. ; $8=$ N.-W. ; $9=$ placette horizontale).

Colonnes 16-19: altitude en $\mathrm{m}$.

Colonne 20: position topographique non encore codée.

Colonnes 21 à 50 et 59 à 80 : à la discrétion du gestionnaire pour noter des critères quaiitatifs ou quantitatifs qu'il codera.

Colonnes 51 à 58 : régénération dans une sous-placette de la placette échantillonnée. La taille moyenne sera indiquée en $\mathrm{cm}$.

Toutes les mesures de la carte 9 sont facultatives. 


\section{ANNEXE 2}

EXEMPLE DE FONCTION MNEMO

FONCTION MNEMO (NESS)

IF (NESS - 9) 510, 511, 512

510 MNEMO $=1$

RETURN

511 MNEMO $=2$

RETURN

512 IF (NESS - 11) 513, 513, 514

513 MNEMO $=3$

RETURN

514 MNEMO $=4$

RETURN

END

Cette fonction transforme les codes discontinus $01,09,11,30$ correspondant aux quatre essences (chênes divers, hêtre, charme, feuillus divers) en un indice MNEMO variant de 1 à 4. 